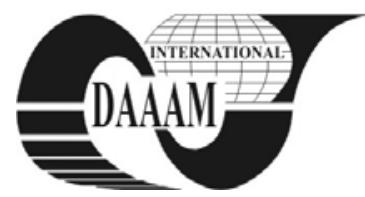

Annals of DAAAM for 2011 \& Proceedings of the 22nd International DAAAM Symposium, Volume 22, No. 1, ISSN $1726-9679$ ISBN 978-3-901509-83-4, Editor B. Katalinic, Published by DAAAM International, Vienna, Austria, EU, 2011 Make Harmony between Technology and Nature, and Your Mind will Fly Free as a Bird Annals \& Proceedings of DAAAM International 2011

\title{
MODIFICATION OF A ZEISS GAUGE BLOCK INTERFEROMETER
}

\author{
KATIC, M[arko]; SIMUNOVIC, V[edran] \& BARSIC, G[orana]
}

\begin{abstract}
Traceability of physical length to the SI unit of meter is realized by means of interferometry, and primary interferometers are used at the highest accuracy levels to perform calibration of national gauge block standards. These devices are very expensive, given the fact that they are manufactured on order and used almost exclusively by national measurement institutes. Therefore, LFSB decided to retrofit and modify an existing Zeiss gauge block interferometer, and preliminary measurements show substantial improvements in accuracy.

Key words: gauge block calibration, traceability, optical interferometry, absolute length measurement, interferogram analysis
\end{abstract}

\section{INTRODUCTION}

For several decades length (meter) has been defined by wavelength of radiation sources in vacuum, first by radiation of Krypton 86 and currently by radiation of Iodine stabilized Helium- Neon laser (CGPM, 1983). Since such definition is not "tangible" and is therefore very impractical to use in everyday measurements, comparison of radiation source wavelength to physical length of an artifact has been done for many years using the principles of interferometry. The use of interferometers to accomplish this task is in principle simple and well defined, but in order to perform highest accuracy measurements a lot of factors have to be carefully considered. Because of high cost and low availability of top level gauge block interferometers, LFSB decided to upgrade its Zeiss interferometer, which used Krypton and Helium spectral lamps, to operate with the current definition of metre.

\section{ZEISS INTERFEROMETER}

Zeiss interferometer (Figure 1) is based on Kosters design (Hariharan, 2003) which performs amplitude splitting of the source radiation (visible light in this case) by a beam splitter which divides the incoming beam into two beams- reference and measurement beam. These two beams are reflected and then recombined at the beam splitter, where they interfere. Zeiss interferometer features a monochromator, based on Kosters prism, which is used to isolate certain frequencies of source radiation- originally Krypton and Helium spectral lamps.

\section{MODIFIED ZEISS INTERFEROMETER}

In order to achieve current state of the art accuracy and measurement uncertainty, several modifications have been done on the previously described Zeiss interferometer. These modifications, described in the following sections, enable the use of lasers as sources of radiation; improve temperature, pressure and humidity monitoring; and enable digital interferogram acquisition and evaluation. Internal optical system was not modified, due to extremely delicate alignment of optical components and sealed optical path.

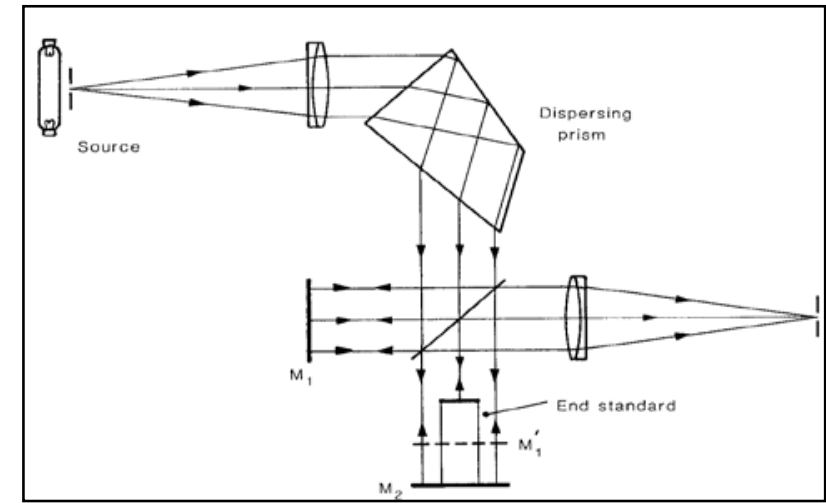

Fig. 1. Zeiss interferometer schematic

\subsection{Laser coupling}

In order to use a laser as the source of radiation, an optical coupling system had to be designed. To achieve flexibility in the design, optical fibers were selected for laser light delivery rather than mirrors. The use of optical fiber requires a fiber launch system (Figure 2) to couple laser light into the fiber. Fiber launch system is comprised of a precision six axis alignment platform, a translation stage for focus adjustment, and a microscope objective. Since multiple wavelength interferometry is used (Malacara, 2005), a multimode cable was chosen. In order to eliminate speckle pattern at the output of the fiber, a rotating diffuser was used to reduce spatial coherence of the laser.

Optical system for interferometer input aperture was designed with achromatic elements to allow for different source wavelengths. Since the focal length and diameter of collimating optics inside the interferometer was not known precisely, a telescope assembly was designed at the input aperture (Figure 3 ) in order to find the correct input numerical aperture.

These modifications resulted in substantial reductions of necessary laser power, due to good coupling efficiency. Furthermore, better alignment of interferometer's optical axis is now possible, which has beneficial effects on measurement uncertainty.

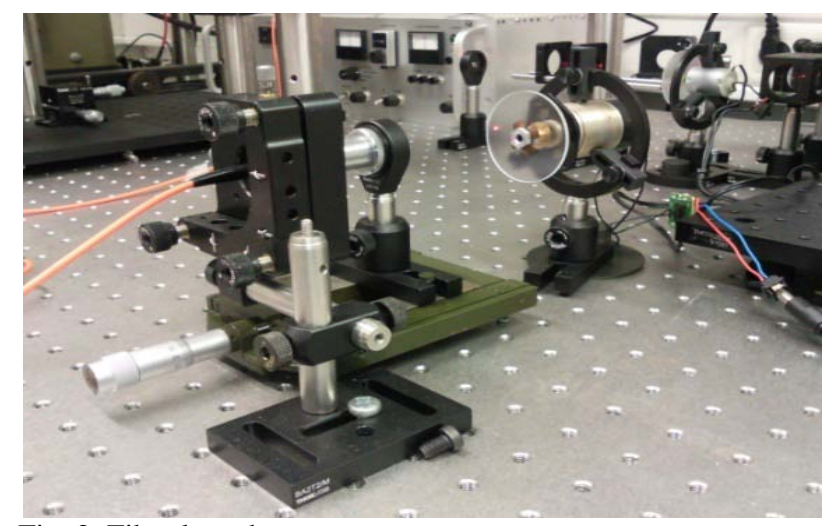

Fig. 2. Fiber launch system 


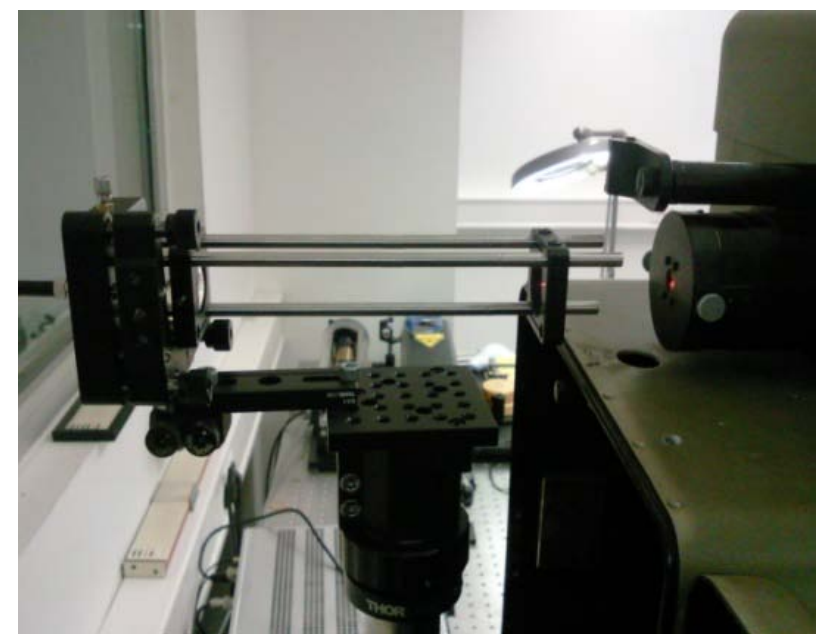

Fig. 3. telescope assembly at interferometer aperture

\subsection{Interferogram acquisition and evaluation}

The acquisition of interferograms is performed digitally with a color CMOS camera. An objective lens was chosen to match the decollimating optics of the interferometer. The camera has a resolution of 5 Megapixels, which provides more than adequate pixel density for fringe fraction analysis.

Interferometric gauge block measurement was previously performed by visual inspection of interferograms with approximately $\lambda / 10 \quad(\sim 60 \mathrm{~nm})$ accuracy. To improve measurement accuracy a software for interferogram acquisition and evaluation was developed (Figure 4).

Its basic purpose is to capture the interferogram from the camera, apply environmental corrections (Birch \& Down, 1994), evaluate fringe fractions automatically, and finally calculate the gauge block length and deviation from nominal length. Measurements of gauge block temperature and air pressure, temperature and humidity are taken at the same time as the interferogram, providing the most accurate information about these conditions at the time of the measurement.

Fringe fractions are determined by an edge detection algorithm developed at LFSB (Katic et al, 2010), which transforms the captured color image into grayscale by applying adaptive threshold based on several histogram traces. The image is then binarized and fringe centers are calculated by least squares fitting. This procedure was found to be accurate to more than $\lambda / 1000(<1 \mathrm{~nm})$, and represents the largest single contribution to improvement of measurement accuracy. Also, synchronous acquisition of environmental measurements further adds to the achieved increase of accuracy.

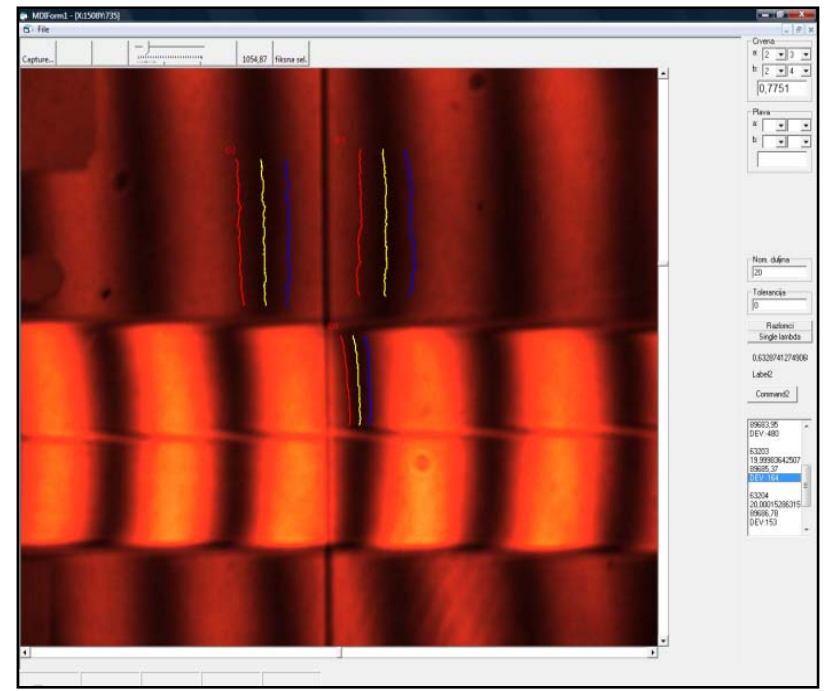

Fig. 4. Interferogram analysis software

\section{RESULTS}

The primary goal was to introduce laser as the wavelength source, and to keep the laser-interferometer coupling system flexible enough to allow the use of laser in other systems. Secondly, interferogram acquisition was substantially improved by use of high resolution digital camera and quality objective. The outcome of these particular modifications can be seen in Figure 5, with evident increase in interferogram quality.

The software that was developed to acquire and analyze interferograms allowed a significant increase in measurement resolution and consequently in measurement accuracy. The fact that environmental corrections are performed simultaneously with interferogram acquisition allows further reduction of associated measurement uncertainty.

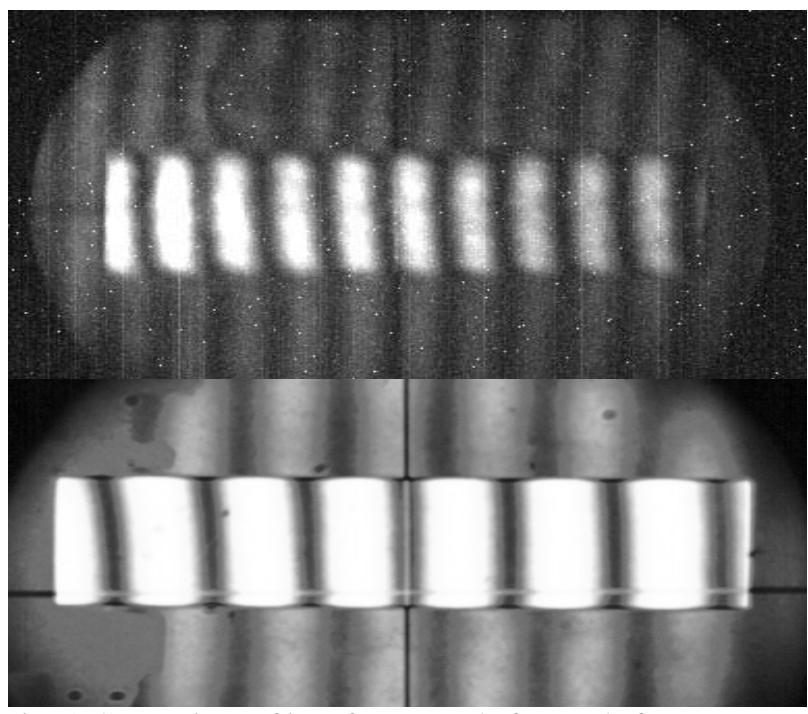

Fig. 5. Comparison of interferograms before and after modifications

\section{CONCLUSION}

Extensive modifications were performed on a Zeiss interference comparator and presented in this paper, including laserinterferometer coupling with speckle reduction, optical system for acquisition of interferograms, and software for interferogram evaluation. It was shown that it is possible to realize competitive performance levels by using only widely available parts and with relatively low costs. The performance of the interferometer is to be confirmed by extensive measurements and intercomparisons with other European NMI's, and will be presented in future works.

\section{REFERENCES}

Resolution 1 of the 17th meeting of the CGPM (1983)

Hariharan, P. (2003), Optical Interferometry, Elsevier Science, ISBN 0-12-311630-9, UK

Malacara, D. (2005), Interferogram analysis for optical testing, CRC Press, ISBN 1-57444-682-7, USA

Birch, K. P.; Downs, M. J. (1994), Correction to the updated Edlen equation for the refractive index of air, Metrology v.31, p.315-316,

Katic, M.; Mudronja, V. \& Simunovic, V. (2010). Edge Uncertainty in Fringe Analysis, Annals of DAAAM for 2010 \& Proceedings of the 21st International DAAAM Symposium, 20-23rd October 2010, Zadar, Croatia, ISSN 1726-9679, ISBN 978-3-901509-73-5, Katalinic, B. (Ed.), pp. 1277-1278, Published by DAAAM International Vienna, Vienna 